\title{
EDITORIAL
}

\section{MilkyWay-2: back to the world Top 1}

\author{
Xiangke LIAO (凶) $)^{1,2}$ \\ 1 Science and Technology on Parallel and Distributed Processing Laboratory, \\ National University of Defense Technology, Changsha 410073, China \\ 2 College of Computer, National University of Defense Technology, Changsha 410073, China
}

(c) Higher Education Press and Springer-Verlag Berlin Heidelberg 2014

On the 41st Top500 list announced in June 2013, the MilkyWay-2 system produced by National University of Defense Technology (NUDT) in China won the first place with a LINPACK test result of 33.86 PFLOPS. It has been one and a half year since its predecessor, MilkyWay-1(TH-1), reached the same place for the first time. On the newest Top500 list published in November 2013, MilkyWay-2 continued to win the champion.

MilkyWay-2 is a milestone achievement supported by the first phase of the National High Technology Program (863 program) "High efficient computer development". It is deployed in the National Supercomputer Center in Guangzhou (NSCC-GZ) and will provide an open platform for research, education and high performance computing service. MilkyWay-2 obtains a series of technical innovations, such as neo-heterogeneous architecture based on similar instruction set, domestic high performance processor, domestic $\mathrm{TH}$ Express 2 network, high density package, high reliability and scalability. It establishes a firm foundation for the ultimate goal of the program, building a 100PFLOPS supercomputer by the year of 2015 .

To introduce the development and technical features of MilkyWay-2, Frontiers of Computer Science releases this special section, which consists of our papers. Liao et al. first provide an overall introduction to MilkyWay-2, with detailed description of its software and hardware systems, key architectural features and application development. The other three papers focus on several key technical innovations of

Received February 19, 2014

E-mail:xkliao@nudt.edu.cn
MilkyWay-2 respectively, including interconnection network, storage system, and fault-tolerant management system. Pang et al. describe the high performance interconnect network of MilkyWay-2, which adopts a hardware/software codesign methodology in order to provide efficient and closeto-hardware communication performance. $\mathrm{Xu}$ et al. give the design of storage subsystem of MilkyWay-2, including its hybrid hierarchy hardware architecture and a parallel file system named H2FS. Finally, Lu et al. depict the fault-tolerant management system, in which they introduce the concept of autonomous management. That is, the computer itself, rather than manpower, takes charge of the fault management work, so that the cost of fault handling reduces from several hours to a few seconds.

The second phase of the MilkyWay-2 program is ongoing now, which plans to build a supercomputer with peak performance over 100PFLOPS, LINPACK efficiency more than $60 \%$ and energy efficiency more than 5GFLOPS/watt. It will be an important technical exploration for Exa-FLOPS computing in the future.

Finally, it should be noticed that, although Chinese HPC obtains fairly good achievements in recent years, even a leading place in some techniques, there is still a big gap in fundamental and key technologies between China and the western world. At present, plenty of high performance projects from all over the world have been proposed, which realizingly take Exa-FLOPS computing as a strategical goal and plan to achieve it in 5-10 years. HPC research is like rowing upstream, not to advance is to drop back. The Chinese HPC community still has a lot of technical challenges to conquer, to keep up with the international Exa-FLOPS competition. 


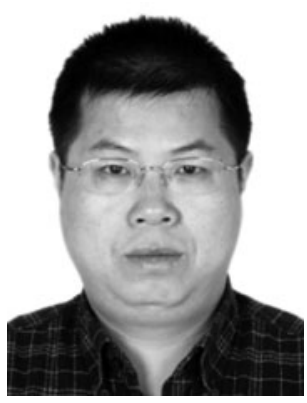

Xiangke Liao received the BS in computer science from Tsinghua University, China and MS degree in computer science from the National University of Defense Technology (NUDT), China. Currently he is a professor at NUDT. His research interests include high performance computing system, operating system, parallel software. He is the chief designer of MilkyWay-2 system. 